\title{
Sexually transmitted infections: where are we now?
}

\author{
C Robertson A Jayasuriya P S Allan
}

J R Soc Med 2007; 100:414-417

This is the third in a series of articles on sexuality and sexual health.

\section{INTRODUCTION}

In 2001 the UK's National Strategy for Sexual Health and HIV was launched; however, the number of diagnoses of sexually transmitted infections (STIs) in the UK has continued to rise. This trend has been driven by ongoing high-risk sexual behaviour and poor access to services, in particular fast access to genitourinary medicine (GUM) clinics. The incidence of infectious syphilis, previously sustained at a low level for years, has dramatically increased. New threats such as lymphogranuloma venereum (LGV) are appearing on the scene. In the face of this, it has become of increasing importance to adopt new screening and prevention strategies, improved diagnostics and ongoing surveillance. Some epidemiological developments and promising strategies for dealing with the STI epidemic are discussed here.

\section{CHLAMYDIA}

Genital Chlamydia trachomatis infection is the most common STI in the UK. The number of uncomplicated chlamydia diagnoses in GUM clinics has risen steadily since the mid1990s, with the highest rates seen in young people. ${ }^{1}$ Up to $80 \%$ of women and $50 \%$ of men may be asymptomatic, hence unrecognized infection is fuelling transmission in the community. The National Chlamydia Screening Programme (NCSP) commenced in England in 2002 with 10 opportunistic screening programmes, with an additional 16 introduced in 2004. ${ }^{2}$ Its aim is to implement and monitor opportunistic screening for genital chlamydia infection for young men and women under 25 years of age. Screening takes place in both health care settings, such as contraception clinics and general practice, and non-health care settings, such as colleges and military bases. Nucleic acid amplification testing (NAAT) is performed on selfcollected vulvovaginal swabs (preferred method of collection for women) or first-catch urine (men and some women). The annual report for 2005 showed an overall

Department of Genitourinary Medicine, Coventry and Warwickshire Hospital, Stoney Stanton Road, Coventry CV1 4FH, UK

Email: ashini.jayasuriya@coventrypct.nhs.uk positivity rate of $11 \% .^{3} \mathrm{~A}$ full screening programme will begin across England in 2007. The challenge will be to ensure the target population is being reached, that adequate counselling and partner notification facilities are in place, and to determine whether this widespread strategy is effectively reducing infection rates.

\section{NUCLEIC ACID AMPLIFICATION TESTS}

NAATs are DNA amplification-based tests. They are highly sensitive and specific, and have allowed self-collected genital specimens to be used for diagnosis of genital Neisseria gonorrhoeae and C. trachomatis. This greatly increases the feasibility and volume of screening outside clinic settings, which is important in improving access to care and reducing waiting times for testing for those who are asymptomatic. In addition, self-sampling is often more acceptable to individuals than the invasive specimen collection required for culture and immunoassays. This development is being used in some GUM clinics in the introduction of 'one-stop clinics' for asymptomatic individuals, where a self-collected vulvovaginal swab (women) or first-pass urine specimen (men) can be tested for gonorrhoea and chlamydia using NAATs, and blood sampled for syphilis and HIV. The specificity of NAATs is not $100 \%$ and confirmation by culture of a NAAT-positive result for gonorrhoea is advisable. ${ }^{5}$ NAATs are also currently unlicensed for rectal or oropharyngeal samples.

\section{GONORRHOEA}

Gonorrhoea remains the second most common bacterial STI in the UK. Infection is easily treatable with appropriate antimicrobials; however, reduced sensitivity and resistance to several classes of antimicrobials has developed and is increasing. Fluoroquinolones were a first-line therapy up until 2003, ${ }^{6}$ when data from the Gonococcal Resistance to Antimicrobials Surveillance Programme (GRASP), a sentinel surveillance study by the Health Protection Agency, showed an increase in ciprofloxacin resistance and a change in the national guidelines was initiated. 2004 data from GRASP showed $N$. gonorrhoeae resistance to ciprofloxacin of $14.1 \%$, penicillin-resistance of $11.2 \%$ and tetracycline resistance of $44.5 \% .{ }^{6}$ Current treatment guidelines recommend cephalosporins (ceftriaxone or cefixime) as first-line therapy. ${ }^{5}$ No isolates demonstrated 
reduced susceptibility to either cephalosporin in $2004{ }^{6}$ however, there are emerging reports of reduced susceptibility to these drugs. ${ }^{7}$ Alternative therapies may be used depending on local antimicrobial sensitivity patterns. These data reinforce the need to confirm any positive NAAT for $N$. gonorrhoeae by culture and testing for antimicrobial sensitivity, and culture remains the diagnostic method of first choice in GUM clinics in the UK. ${ }^{5}$ The other advantage to this is that microscopy of a Gram-stained genital specimen (from the urethra, cervix or rectum) can take place prior to culture, allowing visualization of N. gonorrhoeae as Gram-negative intracellular diplococci in polymorphonuclear leukocytes.

\section{SYPHILIS}

Historically, diagnoses of infectious syphilis at GUM clinics have fluctuated due to changes in sexual behaviour, social changes and war. Diagnoses in the UK peaked sharply towards the end of World War II, followed by a modest reduction, with diagnoses in men then increasing throughout the 1960s and 1970s. Diagnoses of syphilis, particularly in men, declined in the 1980s following emerging awareness of HIV and subsequent behavioural changes. A low prevalence and incidence of infectious (primary, secondary and early latent) syphilis was evident until the late 1990s, when an outbreak of infectious syphilis was reported in Bristol. ${ }^{8}$ Outbreaks were subsequently seen in Manchester (1999), London, Brighton, Nottingham and Newcastle-upon-Tyne. Enhanced surveillance for infectious syphilis has been in place throughout England and Wales since 2002. ${ }^{9}$ In the UK between 1998 and 2004, rates of diagnosis of infectious syphilis in men increased by $1520 \% .{ }^{10}$ The burden of new diagnoses has largely been seen in men who have sex with men (MSM). The median age of cases was 35 years and the vast majority were of white ethnicity. ${ }^{9}$ Oral sex was an important transmission route, with almost half of MSM reporting this as their perceived risk. Significantly, a high percentage of HIV coinfection was reported.9,11 This suggests that the epidemiology of syphilis has been influenced by developments in the HIV epidemic, along with an increase in unsafe sex in both MSM (saunas, cruising areas and the internet) and heterosexuals (commercial sex workers, travel abroad). This behavioural pattern has meant that many partners are anonymous and untraceable, further fuelling the epidemic. Increased incidence within the heterosexual population is an emerging public health problem, with cases of congenital syphilis emerging.

\section{LYMPHOGRANULOMA VENEREUM}

LGV, caused by C. trachomatis serovars L1, L2 or L3, may present as a small papule at the site of infection which progresses to a painless genital ulcer, inguinal lymphadenopathy, fever and proctitis. It is endemic in areas of Africa, India, the Caribbean and Central and South America. Since December 2003, outbreaks have been reported in Western Europe (including the Netherlands, from which the original reports came, and the UK) and more recently from the USA. These have been concentrated in sexual networks consisting of MSM (mostly white and HIV-positive) in large cities. ${ }^{12,13}$ Most have anorectal symptoms (such as discharge, pain, tenesmus, and bloody stools), or proctitis. Systemic symptoms such as fever and malaise may occur. Swollen painful inguinal nodes and penile discharge have also been reported; however, some are asymptomatic. If left untreated, perirectal fistulae, abscesses, strictures and scarring may result. The primary papule often goes unnoticed.

In October 2004, the UK's Health Protection Agency launched an initiative to raise awareness and improve case ascertainment in the UK, and by the end of March 2006 341 cases had been confirmed. ${ }^{14}$ Cases have mimicked Crohn's disease ${ }^{15}$ and other non-infectious gastrointestinal diseases, hence vigilance and enquiring about sexual history in specialties outside genitourinary medicine is important. LGV has also mimicked primary genital herpes ${ }^{16}$ and syphilis.

Diagnosis is through C. trachomatis detection by NAAT (although currently unlicensed for rectal specimens), which should be confirmed by the laboratory using polymerase chain reaction, followed by detection of LGV serovars using molecular methods. ${ }^{12}$ Cell culture can also be used. If a case is suspected, liaising with local microbiology laboratories is important so that correct samples can be taken and forwarded and analysed as appropriate. The most common treatment regimen recommended is a three-week course of oral doxycycline. ${ }^{17}$ Chronic infection, including fistulae and strictures, often necessitates surgical intervention.

\section{HUMAN PAPILLOMAVIRUS}

Over 30 types of human papillomavirus (HPV) can be sexually transmitted. Exophytic anogenital warts may result, but many individuals with detectable genital tract HPV will have no clinically apparent lesion. In spite of this, anogenital warts are the most frequently diagnosed viral STI in the UK, with almost 80,000 new diagnoses between 2003 and 2004. ${ }^{18}$ HPV 6 and 11 account for approximately $90 \%$ of anogenital warts, ${ }^{19}$ and HPV types 16 and 18 are responsible for more than $70 \%$ of cervical squamous cell carcinoma. ${ }^{20}$ There are currently numerous vaccines at different stages of development directed against HPV. Two vaccines are currently undergoing phase III trials, one developed by GlaxoSmithKline (a bivalent vaccine against HPV types 16 and 18) and one developed by Merck (a 
quadrivalent vaccine against HPV types 6, 11, 16 and 18). Merck's vaccine, marketed as Gardasil, was licensed in the UK in late 2006, although is not as yet available on the NHS. Both vaccines comprise papillomavirus-like particles (consisting of the viral capsid without the DNA) which are thought to induce production of neutralizing antibodies, thus protecting against HPV and ultimately the development of cervical intraepithelial neoplasia, other genital premalignant lesions, and invasive cervical carcinoma. Both vaccines in development are administered in three doses over a six-month period. ${ }^{21}$ Recent data suggest that the HPV vaccine is highly immunogenic, with 98\% seropositivity for HPV types 16 and 18 antibodies maintained at 4.5 years. ${ }^{22}$ The safety profile of the vaccine is good, with the main adverse reaction being pain at the injection site. ${ }^{21}$ Merck's intention-to-treat analysis suggested a reduction in the risk of precancerous cervical changes or non-invasive cancer by $97 \%$ over an average two-year follow-up period. Although results so far look promising, long-term data are needed, particularly given the natural history of the development of cervical intraepithelial neoplasia (CIN) and invasive cervical carcinoma, with high-grade CIN taking roughly 10 years to progress to invasive carcinoma.

In order for an HPV vaccine to be effective on both the individual and the population level, it would need to be given to children or adolescents in an effort to confer immunity before individuals become sexually active. Research from the UK showed that a quarter of women and almost a third of men became sexually active before the age of 16 years, with a not insignificant minority becoming sexually active before the age of 13 years. ${ }^{24}$ It is also unclear as yet whether it would be recommended for both men and women, although one mathematical model suggests that vaccinating both young men and women will have more of an impact on cases of cervical dysplasia and invasive cancer. ${ }^{25}$ Concern has also been voiced by some groups that HPV vaccination would lead to an adverse effect on adolescent sexual behaviour, which would need to be learned through monitoring groups. Clearly a vaccination programme will not eliminate the need for cervical cancer screening; it will remain essential to detect any problems in women who have not been vaccinated or are already infected with HPV, and those infected by HPV strains not covered by the vaccine.

\section{CONCLUSION}

The public health white paper Choosing Health: Making Healthier Choices Easier ${ }^{26}$ set a number of targets for 20052008 regarding sexual health. These include rollout of the NCSP as previously described, access to a GUM clinic appointment within 48 hours by 2008, and a reduction in the rates of STIs. Meeting these objectives will be challenging, as evidenced above. The HIV epidemic is also integral to this, as STIs, particularly those causing genital ulcerative disease, facilitate HIV transmission. Community and clinician awareness of the range of presentations of STIs is essential, often provided by local initiatives tailored to that population along with broader advice from the Department of Health. Community-based facilities and initiatives and those based outside the clinic setting are likely to have an increasing role.

Competing interests $\mathrm{CR}, \mathrm{AJ}$ and PSA have no conflicts of interest.

Guarantor AJ.

Contributorship All authors contributed equally to this work.

\section{REFERENCES}

1 www.hpa.org.uk/infections/topics_az/hiv_and_sti/sti-chlamydia/ epidemiology (accessed April 2006)

2 www.dh.gov.uk/PolicyAndGuidance/HealthAndSocialCareTopics/ SexualHealth/SexualHealthGeneralInformation/

SexualHealthGeneralArticle/fs/ en?CONTENT_ID=4084098\&chk+CSLxsK (accessed April 2006)

3 Department of Health. The Annual Report of the National Chlamydia Screening Project in England, 2004/05. London: DoH, November 2005. www.dh.gov.uk (accessed April 2006)

4 Katz AR, Effler PV, Ohye RG, et al. False-positive gonorrhoea test results with a nucleic amplification test: the impact of low prevalence on positive predictive value. Clin Infect Dis 2004;38:814-19

5 British Association for Sexual Health and HIV. National Guideline on the Diagnosis and Treatment of Gonorrhoea in Adults 2005. London: BASHH, 2005. www.bashh.org (accessed April 2006)

6 Health Protection Agency. GRASP: The Gonococcal Resistance to Antimicrobials Surveillance Programme-Annual Report 2004. London: HPS, August 2005

7 Dr S Allan, personal correspondence, May 2006

8 Simms I, Fenton K, Ashton M, et al. The re-emergence of syphilis in the United Kingdom: the new epidemic phases. Sex Transm Dis 2005;32:220-6

9 Health Protection Agency. Enhanced surveillance of infectious syphilis in England and Wales: data to end of 2005. London: HPA, April 2006. www.hpa.org.uk/cdr/pages/hiv_STIS.htm (accessed April 2006)

10 Health Protection Agency. Epidemiological Data-Syphilis. London: HPA, November 2005. www.hpa.org.uk/infections/topics_az/ hiv_and_sti/sti-syphilis/epidemiology/epi (accessed April 2006)

11 Cohen CE, Winston A, Asboe D, et al. Increasing detection of asymptomatic syphilis in HIV patients. Sex Transm Infect 2005;81:217-9

12 Health Protection Agency. STBRL Newsletter. London: HPA, Spring 2005

13 Health Protection Agency. CDR Weekly: News. London: HPA, 2005; 15(30)

14 www.aidsalliance.org (accessed May 2006)

15 Forrester B, Pawade J, Horner P. The potential role of serology in diagnosing lymphogranuloma venereum (LGV): a case mimicking Crohn's disease. Sex Transm Infect 2006;82:139-40

16 Niewenhuis RF, Ossewaarde JM, van der Meijden WI, et al. Unusual presentation of early lymphogranuloma venereum in an HIV-1 infected patient: effective treatment with $1 \mathrm{~g}$ azithromycin. Sex Transm Infect 2003;79:453-5 
17 British Association for Sexual Health and HIV. 2001 National Guideline for the Management of Lymphogranuloma Venereum (LGV). London: BASHH, 2001. www.bashh.org (accessed April 2006)

18 www.bma.org/ap.nsf/content/stiupd06 (accessed April 2006)

19 Pattman R, Snow M, Handy P, et al. Oxford Handbook of Genitourinary Medicine, HIV, and AIDS. Oxford: Oxford University Press, 2006

20 Munoz N, Bosch PX, Castellsagne X, et al. Against which human papillomavirus types shall we vaccinate and screen? The international perspective. Int J Cancer 2004;111:278-85

21 Steinbrook R. The potential of human papilloma virus vaccines. NEJM 2006;354:1109-12

22 Harper DM, Franco EL, Wheeler CM, et al. Sustained efficacy up to 4.5 years of a bivalent L1 virus-like particle vaccine against human papillomavirus types 16 and 18: follow-up from a randomised control trial. Lancet 2006;367:1247-55

23 www.merck.co./newsroom/press_releases/research_and_development/ 2005_1006.html (accessed May 2006)

24 Wellings K, Nanchahal K, Macdowall W. Sexual behaviour in Britain: early heterosexual experience. Lancet 2001;358:1843-50

25 French KM, Barnabas RV, Garnett GP, et al. Strategies for vaccinating against human papillomavirus. Nottingham: BASHH Spring Meeting, May 2006

26 Department of Health. Choosing Health: Making Healthier Choices Easier. London: DoH, 2004. www.dh.gov.uk/PublicationsAndStatistics/ Publications/PublicationsPolicyAndGuidanceCONTENT_ID= 4094550\&Chk $=\mathrm{aN5Cor}$ (accessed April 2006)

\section{International Journal of STD \& AIDS Clinical Practice in Sexual Health Official journal of BASHH and IUSTI}

If you are involved in researching and treating sexually transmissible infections, HIV and AIDS, then the International Journal of STD and AIDS is the journal for you.

This peer-reviewed monthly journal contains in-depth editorial reviews, original articles and research, short papers, case reports, audit reports and CPD papers. There is also a lively correspondence column, a book review section, and news from the genitourinary medicine associations. Occasional supplements are also published and are supplied free to subscribers.

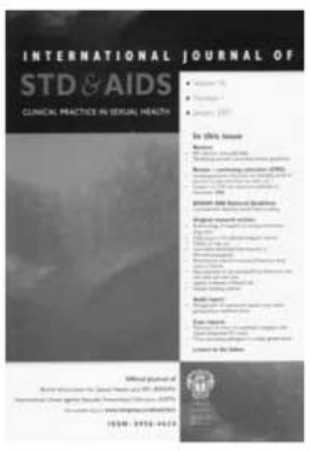

TO FIND OUT FURTHER INFORMATION OR TO VIEW A FREE SAMPLE ISSUE VISIT www.rsmpress.co.uk/std.htm 